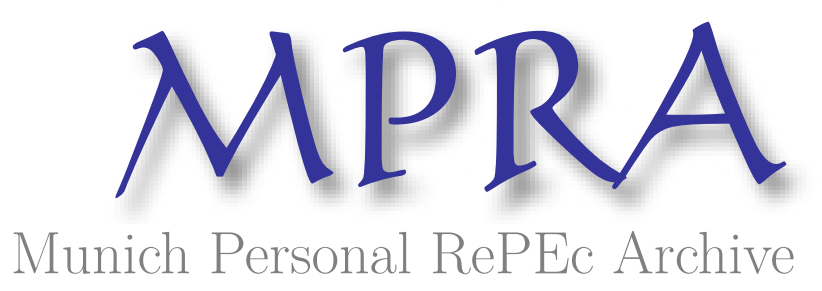

\title{
Ascendant altruism and asset price bubbles
}

Bosi, Stefano and Ha-Huy, Thai and Pham, Cao-Tung and

Pham, Ngoc-Sang

EPEE, Université Paris-Saclay, EPEE, Université Paris-Saclay;

TIMAS, Thang Long University, EPEE, Université Paris-Saclay, EM

Normandie Business School, Métis Lab

1 November 2021

Online at https://mpra.ub.uni-muenchen.de/110522/

MPRA Paper No. 110522, posted 09 Nov 2021 11:55 UTC 


\title{
Ascendant altruism and asset price bubbles*
}

\author{
Stefano BOSI ${ }^{\dagger}$ Thai HA-HUY ${ }^{\ddagger}$ Cao-Tung PHAM $^{\S}$ \\ Ngoc-Sang PHAM $\mathbb{1}$
}

November 1, 2021

\begin{abstract}
We consider an overlapping generations economy with altruism towards parents and a long-lived asset that delivers no dividends (pure bubble asset). We explore the role of ascendant altruism on the dynamics properties of equilibrium and rational bubbles in the cases of exogenous and endogenous growths.
\end{abstract}

JEL classification codes: C61, D64, E44, G10.

Keywords: Overlapping generations, ascendant altruism, capital accumulation, growth, rational bubbles.

\section{Introduction}

In The Theory of Moral Sentiments (1759), Smith departed from Hume's utilitarian notion of sympathy, that is the current welfare-maximization approach, by observing that altruism does not stem from original sensations but from sympathetic images of these sensations. ${ }^{1}$ Becker $(1974,1976)$ introduced instead an "egocentric" perspective considering a parents' utility which includes the utility of offsprings, while Axelrod (1984) went back to Hume's "egoistic" view. From a formal point of view, the hypothesis of descendant altruism à la Becker bridges the overlapping generations (hereinafter, OLG) and the infinite-horizon literatures since the Barro's seminal contribution (Barro, 1974).

${ }^{*}$ We would like to thank an anonymous referee for her(his) very thoughtful comments and suggestions which have helped us to substantially improve our paper. Stefano Bosi and Thai Ha-Huy acknowledge the financial support from the Agence Nationale de la Recherche (FIRE project, grant number ANR-15-CE33-0 0 01; LABEX MME-DII, grant number ANR-11-LBX-0023-01). The authors have no conflicts of interest to declare.

${ }^{\dagger}$ Corresponding author. EPEE, Université Paris-Saclay. Email: stefano.bosi@universite-parissaclay.fr.

${ }^{\ddagger}$ EPEE, Université Paris-Saclay; TIMAS, Thang Long University. Email: thai.hahuy@univ-evry.fr.

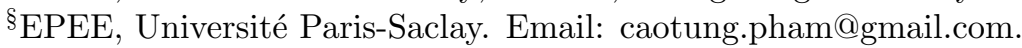

${ }^{\mathbb{E}}$ EM Normandie Business School, Métis Lab. Email: npham@em-normandie.fr.

1 "Every man feels his own pleasures and his own pains more sensibly than those of other people. The former are original sensations; the latter the reflected or sympathetic images of those sensations. The former may be said to be the substance, the latter the shadow." (Smith, 1759). 
Although there is a huge literature on the descendant altruism from parents to children (see Michel et al. (2006) for a review), very few studies investigate the effects of ascendant altruism (from children to parents). On the other hand, the question of financial asset bubble has been recently revisited by many scholars because it may have important effects not only on the financial markets but also on the real economy. ${ }^{2}$ Motivated by these two observations, our paper aims to examine the effects of ascendant altruism on the dynamics of asset price bubble and capital accumulation.

To address these issues, we introduce two ingredients in an OLG model à la Diamond (1965): (1) the ascendant altruism from children to parents, and (2) a pure bubble asset (which delivers no dividend) à la Tirole (1985). ${ }^{3}$ The ascendant altruism in our model is modeled by a direct transfer from children to parents ("upstream transfer" ). ${ }^{4}$ Several empirical studies (Cai et al., 2006; Kazianga, 2006; Nguyen et al., 2012; Park, 2014) show the presence of these upstream transfers (although there are several reasons of upstream transfers, altruism remains an important motive) especially in countries whose public old-age insurance is not well-developed and parents' income is quite low. ${ }^{5}$

Our first contribution is to explore the interplay between ascendant altruism and asset price bubble in an exogenous growth model. As usual, we say that there is a bubble if the equilibrium price of the above asset is strictly positive. In his seminal article, Tirole (1985) shows that in an OLG model, there exists an equilibrium with bubble only if the interest rate at the steady state of the Diamond economy (so-called benchmark interest rate) is lower than the population growth rate (the low interest rate condition). In our model with ascendant altruism, we obtain a generalized version of this result: bubbles exist only if the so-called modified interest rate (i.e., the interest rate in the economy with ascendant altruism and golden rule capital stock but without bubble) is lower than the population growth rate.

The modified interest rate may not be monotonic in the ascendant altruism degree because the altruism generates two effects: the income when young is decreasing in $y$ but the income when old is increasing in $y$. So, our low interest rate condition leads to important implications. Let us mention two of them. First, if the elasticity of intertemporal substitution (EIS, for short) is less than 1, there exists a steady state with bubble if and only if the benchmark interest rate is lower than the population growth rate and the ascendant altruism is moderate (in the sense that it is lower than an exogenous threshold); in this case, the value of bubble is decreasing in the altruism degree. The intuition is that when the EIS is less than 1, the total savings is decreasing in the ascendant altruism degree. Since agents use part of the total savings to finance the purchase of bubble asset, the ascendant altruism must not be too high.

Second, if the EIS is higher than 1, a bubble may exist even the benchmark interest rate is higher than the population growth rate. This scenario represents an important

\footnotetext{
${ }^{2}$ The reader can also find excellent surveys in Brunnermeier and Oehmke (2013), Miao (2014) and Martin and Ventura (2018).

${ }^{3}$ For a model with descendant altruism and bubble, the reader is referred to Bosi et al. (2018b) and Clain-Chamosset-Yvrard and Seegmuller (2019).

${ }^{4}$ The ascendant altruism in our paper is based on a moral constraint which is comparable to the legal constraint of a Pays-As-You-Go (PAYG) pension scheme.

${ }^{5}$ The reader is referred to Laferrere and Wolff (2006) for an excellent survey on upstream transfers.
} 
difference between our framework and that of Tirole (1985). The basic reason is that there exists a steady state with bubble if and only if that the modified interest rate (but not the benchmark interest rate) is low. In this scenario, the modified interest rate can be lower than the population growth rate (that is lower than the benchmark interest rate) if the ascendant altruism is strong enough. This in turn ensures the existence of bubble. Moreover, the value of bubble is increasing in the ascendant altruism degree.

Our second contribution is to investigate the role of ascendant altruism in an endogenous growth model à la Romer (1986). We show that the Balanced Growth Path (BGP) is a unique equilibrium. When the EIS $\leq 1$, we find that ascendant altruism affects the portfolio composition: not only it reduces the portfolio value (savings) but also the bubble share in total savings along the BGP. However, when the EIS is higher than 1 , the relative size of bubble is increasing in the ascendant altruism degree if the altruism is strong enough.

It should be noticed that different modelings of altruism have different outcomes in terms of occurrence of bubbles. Indeed, Bosi et al. (2019) show that the introduction of altruism à la Barro (transfers from parents to children) in an OLG model à la Tirole (1985) rules out any pure bubble even if the bequests or the share of altruistic agents in total population become arbitrarily small. Bosi et al. (2018b) investigate the effects of descending altruism on the dynamics of bubbles of an assets with non-stationary dividends in an two-period OLG model. Clain-Chamosset-Yvrard and Seegmuller (2019) study the interplay between real estate bubble, capital accumulation and taxation in a three-period OLG model with descending altruism. Although they employ different models, both Bosi et al. (2018b) and Clain-Chamosset-Yvrard and Seegmuller (2019) suggest that a sufficiently high degree of descending altruism may promote a productive real estate bubble.

The rest of the paper is organized as follows. The fundamentals and the economy structure are introduced in Section 2. Section 3 considers the interplay between ascendant altruism and asset bubble in an exogenous growth model while Section 4 consider an endogenous growth model and examines the role of ascendant altruism on the growth rate and the dynamics of bubble. Section 5 concludes. All the technical proofs are gathered in Appendices.

\section{Framework}

We firstly consider a two-period OLG model with rational bubbles in the spirit of Tirole (1985) and Weil (1987).

Production. Assume that there is a representative firm whose technology is represented by a constant returns to scale production function $F(K, L)$ where $K$ and $L$ are the aggregate capital and the labor forces. Assume that $F \in C^{2}$ is strictly increasing and concave. Denote $f(k) \equiv F(k, 1)$ where $k \equiv K / L$ denotes the capital intensity. Profit maximization determines the factor demand:

$$
R_{t}=R\left(k_{t}\right) \equiv f^{\prime}\left(k_{t}\right) \text { and } w_{t}=w\left(k_{t}\right) \equiv f\left(k_{t}\right)-k_{t} f^{\prime}\left(k_{t}\right)
$$

where $R_{t}$ and $w_{t}$ represent the return on capital and the wage rate. This technology is summarized by two key elasticities: the capital share in total income and the elasticity 
of capital-labor substitution:

$$
\alpha=\alpha(k) \equiv \frac{k f^{\prime}(k)}{f(k)} \text { and } \sigma=\sigma(k)=\alpha(k) \frac{w(k)}{k w^{\prime}(k)}
$$

Households. Preferences are rationalized by an additively separable utility function:

$$
u\left(c_{t}\right)+\beta u\left(d_{t+1}\right)
$$

where $u \in C^{2}$ is strictly increasing, concave, $u^{\prime}(0)=\infty$, parameter $\beta \in(0,1)$ represents the degree of patience while $c_{t}$ and $d_{t+1}$ denote the consumption demands at time $t$ and $t+1$ of a household born at time $t$. As above, preferences are also expressed in terms of elasticity:

$$
\varepsilon(c)=-\frac{u^{\prime}(c)}{c u^{\prime \prime}(c)}
$$

is the nonnegative elasticity of intertemporal substitution (EIS, for short) capturing the interplay between income and substitution effects over time.

$N_{t}$ new individuals enter the economy at time $t$. The growth factor of population is supposed to be constant: $n=N_{t+1} / N_{t}$. Each young agent supplies one unit of labor.

Agents save through a portfolio $\left(a_{t}, s_{t}\right)$ of pure bubble asset and physical capital. Consumption prices are normalized to one. $q_{t}$ denotes the asset price in consumption units while $b_{t} \equiv q_{t} a_{t}$ the value of asset. In our model, the pure bubble asset do not bring any dividend. ${ }^{6}$

The household born at date $t$ chooses positive vector $\left(c_{t}, d_{t+1}, s_{t}, a_{t}, h_{t}\right)$ to maximize their utility subject to the following constraints:

$$
\begin{aligned}
c_{t}+s_{t}+q_{t} a_{t}+h_{t} & \leq w_{t} \\
d_{t+1} & \leq R_{t+1} s_{t}+q_{t+1} a_{t}+n h_{t+1} .
\end{aligned}
$$

Inequalities (5a) and (5b) are the first- and second-period budget constraints. We assume that children care about parents. Formally, the terms $h_{t}$ in $(5 \mathrm{a})$ represents transfers from children born at period $t$ to their parents.

Several empirical studies show the existence of this transfer in Burkina Faso (Kazianga, 2006), China (Cai et al., 2006), South Korea (Park, 2014), and Vietnam (Nguyen et al., 2012). For example, Nguyen et al. (2012) focus on the case of Vietnam and find that transfers from children to parents are motivated by the desire to provide old age support and are closely related to the ability of children to give and the needs of parents. ${ }^{7}$ These transfers are present in many countries whole public old-age insurance is not well-developed.

Motivated by this empirical observation, we assume that transfers from children to parents depend on children's consumption. It implicitly means that when young

\footnotetext{
${ }^{6}$ See, for instance, Santos and Woodford (1997), Le Van and Pham (2016), Bosi et al. (2017, 2018a, 2021) for general equilibrium models with bubbles of assets having positive dividends.

${ }^{7}$ More precisely, using the Vietnam Living Standard Survey conducted in 1997/1998, Nguyen et al. (2012) show that monetary transfers from children depend on the needs of parents and the ability of children. On average, $31 \%$ of elderly parents receive transfers from their non-coresiding children. For these receiving parents, transfers from children represent $58 \%$ of per capita household expenditure.
} 
people buy consumption goods (for example, food, car, travel, ...), they think to their parents and give a gift to them (the value of this gift depends on the consumption of children). Formally, we assume that

$$
h_{t}=y c_{t} .
$$

Our modeling of ascendant altruism is different from the conventional one in the literature (Barro, 1974; Michel et al., 2006), where they consider that children's utility function depends on their consumptions and their parents' utility. However, as recognized by Michel et al. (2006), page 1092, this setup (based on utility function) raises some modeling concerns. ${ }^{8}$ Our setup can avoid these concerns.

Definition 1. A positive list $\left(q_{t}, R_{t}, w_{t}, c_{t}, d_{t+1}, h_{t}, s_{t}, a_{t}, k_{t+1}\right)_{t \geq 0}$ is an intertemporal equilibrium for the economy with ascendant altruism if: (1) the allocations $\left(c_{t}, d_{t+1}, h_{t}, s_{t}, a_{t}\right)$ maximize $u\left(c_{t}, d_{t+1}\right)$ given $\left(q_{t}, q_{t+1}, R_{t}, w_{t}, h_{t+1}\right)$ subject to constraints (5a), (5b) and (6), and (2) markets clear:

$$
\text { physical capital: } s_{t}=n k_{t+1} ; \quad \text { financial asset: } a_{t}=n a_{t+1} \text {. }
$$

An equilibrium is said to be bubbly (respectively, bubbleless) if $q_{0}>0$ (respectively, $\left.q_{0}=0\right)$.

\section{Ascendant altruism and bubble}

In the bubbly equilibrium, the consumer's program leads to a no-arbitrage condition:

$$
q_{t} R_{t+1}=q_{t+1} .
$$

So, there is a bubble if and only if $q_{t}>0 \forall t$. Since, in equilibrium, $a_{t}=a_{0} / n^{t}$, condition (8) becomes $b_{t}=\frac{n}{R_{t+1}} b_{t+1}=\frac{n}{f^{\prime}\left(k_{t+1}\right)} b_{t+1}$.

By the no-arbitrage condition, we have $d_{t+1}=R_{t+1}\left(s_{t}+b_{t}\right)+n h_{t+1}$. Since $h_{t}=y c_{t}$, the household's consumption when young and the transfer $h_{t}$ become

$$
c_{t}=\frac{w_{t}-\left(s_{t}+b_{t}\right)}{1+y}, \quad h_{t}=\frac{y\left(w_{t}-\left(s_{t}+b_{t}\right)\right)}{1+y} .
$$

Since $h_{t}=y c_{t}$, the first-order condition for the household maximization problem gives us

$$
u^{\prime}\left(c_{t}\right)=(1+y) \beta R_{t+1} u^{\prime}\left(d_{t+1}\right)
$$

To sum up, the dynamics of intertemporal equilibrium is represented by a threedimensional system

$$
\begin{aligned}
c_{t} & >0, k_{t+1}>0 \\
b_{t} & =\frac{n}{f^{\prime}\left(k_{t+1}\right)} b_{t+1} \\
w\left(k_{t}\right) & =(1+y) c_{t}+n k_{t+1}+b_{t} \\
u^{\prime}\left(\frac{w_{t}-\left(n k_{t+1}+b_{t}\right)}{1+y}\right) & =(1+y) \beta R_{t+1} u^{\prime}\left(R_{t+1}\left(n k_{t+1}+b_{t}\right)+n h_{t+1}\right),
\end{aligned}
$$

\footnotetext{
${ }^{8}$ For example, past variables are given and cannot be modified. So, it is not trivial to understand the significance of a backward dynamics of the capital stock.
} 
where $k_{0}$ is given. So, the total savings $n k_{t+1}+b_{t}$ depends on the income $w_{t}$, the interest rate $R_{t+1}$, the altruism amount $h_{t+1}$ and the altruism degree $y$. Of course, when $y=0$, we recover Tirole (1985).

\subsection{Steady state analysis}

First of all, we look at the steady state. At the steady state, the value of bubble and the physical capital are determined by

$$
\begin{aligned}
& b\left(f^{\prime}(k)-n\right)=0 \\
& u^{\prime}\left(\frac{w(k)-n k-b}{1+y}\right)=(1+y) \beta f^{\prime}(k) u^{\prime}\left(f^{\prime}(k)(n k+b)+n y \frac{w(k)-n k-b}{1+y}\right) .
\end{aligned}
$$

Since the bubbleless steady state is exactly that in the case without financial asset, we focus on the bubbly steady state. Before doing this, we introduce some notations.

Definition 2. Denote $k^{n}$ the unique value satisfying $f^{\prime}\left(k^{n}\right)=n$ and $k^{D}$ the capital level at the steady state of the Diamond economy (economy with neither bubble nor altruism) $k^{D}$ is determined by $u^{\prime}\left(w\left(k^{D}\right)-n k^{D}\right)=f^{\prime}\left(k^{D}\right) \beta u^{\prime}\left(f^{\prime}\left(k^{D}\right) n k^{D}\right) .{ }^{9}$ We call $f^{\prime}\left(k^{D}\right)$ the benchmark interest rate

At the steady state with bubble $(b>0)$, we have $k=k^{n}$ and the value of bubble $b$ is determined by

$$
u^{\prime}\left(\frac{w\left(k^{n}\right)-n k^{n}-b}{1+y}\right)=(1+y) \beta n u^{\prime}\left(n\left(n k^{n}+b\right)+n y \frac{w\left(k^{n}\right)-n k^{n}-b}{1+y}\right) .
$$

First, we precise conditions ensuring the existence of a steady state with bubble $(b>0)$.

Lemma 1. The existence of a steady state with bubble is equivalent to the satisfaction of the two following conditions:

$$
\mathcal{R}(y) \equiv \frac{w\left(k^{n}\right)-n k^{n}}{>} \frac{u^{\prime}\left(\frac{w\left(k^{n}\right)-n k^{n}}{1+y}\right)}{(1+y) \beta u^{\prime}\left(n f^{\prime}\left(k^{n}\right) k^{n}+n y \frac{w\left(k^{n}\right)-n k^{n}}{1+y}\right)}<n .
$$

Proof. See Appendix.

Notice that $\mathcal{R}(y)$ (named the "modified interest rate") can be interpreted as the real interest rate of the economy without bubble and with the capital stock $k^{n}$. Indeed, we can rewrite $\mathcal{R}(y)$ as

$$
\mathcal{R}(y)=\frac{\frac{u^{\prime}\left(\frac{w\left(k^{n}\right)-n k^{n}}{1+y}\right)}{\beta u^{\prime}\left(n f^{\prime}\left(k^{n}\right) k^{n}+n y \frac{w\left(k^{n}\right)-n k^{n}}{1+y}\right)}}{1+y}=\frac{\frac{u^{\prime}(c(y))}{\beta u^{\prime}(d(y))}}{1+y}=\frac{1+r(y)}{1+y} \approx r(y)-y,
$$

\footnotetext{
${ }^{9}$ We implicitly assume here that $k^{D}$ exists and unique.
} 
where $c(y) \equiv \frac{w\left(k^{n}\right)-n k^{n}}{1+y}, d(y) \equiv n f^{\prime}\left(k^{n}\right) k^{n}+n y \frac{w\left(k^{n}\right)-n k^{n}}{1+y}$. So, the existence of bubbly steady state requires that the modified interest rate is lower than the population growth rate. In this sense, condition (13b) can be considered as a generalized version of the low interest rate condition (capital over-accumulation) in Tirole (1985) which corresponds to the case when there is no altruism (i.e., $y=0)$. Indeed, if $y=0,(13 \mathrm{~b})$ becomes

$$
\mathcal{R}(0)<n \Leftrightarrow \frac{u^{\prime}\left(w\left(k^{n}\right)-n k^{n}\right)}{\beta f^{\prime}\left(k^{n}\right) u^{\prime}\left(f^{\prime}\left(k^{n}\right) k^{n}\right)}<\frac{u^{\prime}\left(w\left(k^{D}\right)-n k^{D}\right)}{\beta f^{\prime}\left(k^{D}\right) u^{\prime}\left(f^{\prime}\left(k^{D}\right) n k^{D}\right)} .
$$

Under mild assumptions, the function $\frac{u^{\prime}(w(k)-n k)}{\beta f^{\prime}(k) u^{\prime}\left(f^{\prime}(k) k\right)}$ is increasing in $k$. So, we have $k^{n}<k^{D}$, or, equivalently, $f^{\prime}\left(k^{D}\right)<f^{\prime}\left(k^{n}\right)$. This is the low interest rate condition (or over-accumulation) in Tirole (1985).

However, condition $(13 \mathrm{~b})$, i.e., $\mathcal{R}(y)<n$, depends on the ascendant altruism degree $y$ and in general it does not necessarily ensure that $\mathcal{R}(0)<n$ (i.e., $k^{n}>k^{D}$ ) because the function $\mathcal{R}$ may not be monotonic.

In the following result, we show the role of different factors (ascendant altruism, EIS, interest rate of the Diamond economy) on the existence of bubbly steady state and the value of bubble.

Proposition 1. Assume that $u(c)=\frac{c^{1-\frac{1}{\epsilon}}-1}{1-\frac{1}{\epsilon}}, \forall c$, with $\epsilon>0$ (the case $\varepsilon=1$, let $u(c)=\ln (c))$. Assume that the function $\frac{f(k)}{k f^{\prime}(k)}$ is decreasing in $k$ and $w\left(k^{n}\right)-n k^{n}>0 .{ }^{10}$ Let $y>0$.

1. Consider the case where the elasticity of intertemporal substitution $\varepsilon \leq 1$. The existence of a bubbly steady state is equivalent to

$$
\begin{aligned}
& \quad f^{\prime}\left(k^{D}\right)<n \quad(\text { or, equivalently, } \mathcal{R}(0)<n) \\
& \text { and } 0<y<\hat{y},
\end{aligned}
$$

where $\hat{y}$ is the unique solution to $\mathcal{R}(y)=n$.

Moreover, the value of bubble is decreasing in $y$.

2. Consider the case $\varepsilon>1$.

(a) If $f^{\prime}\left(k^{D}\right)<n$ (or, equivalently, $\mathcal{R}(0)<n$ ), then there exist $y$ and $\bar{y}$ with $0 \leq y \leq \bar{y}$ such that the existence of a bubbly steady state is equivalent to either $y<\underline{y}$, or $y>\bar{y}$.

The value of bubble is decreasing in $y$ on the interval $(0, y)$, and increasing in $y$ on the interval $(\bar{y}, \infty)$. Moreover, $\lim _{y \rightarrow \infty} b=w^{n}-\bar{n} k^{n}$.

(b) If $f^{\prime}\left(k^{D}\right)>n$ (or, equivalently, $\mathcal{R}(0)>n$ ), then there exists $\bar{y}$ such that the existence of a bubbly steady state is equivalent to $y>\bar{y}$.

Moreover, the value of bubble is increasing in $y$ and $\lim _{y \rightarrow \infty} b=w^{n}-n k^{n}$.

Proof. See Appendix.

\footnotetext{
${ }^{10}$ These condition hold for a large class of production function, including the Cobb-Douglas function $f(k)=A k^{\alpha}$ with $\alpha<0.5$.
} 
Condition (16a) is a version of the capital over-accumulation or the low interest rate condition in the literature. In Tirole (1985), this condition is essential to have a steady state with bubble. However, it is not essential in our model because of the presence of the ascendant altruism. The added-value of Proposition 1 with respect to the existing literature is to explore the role of the ascendant altruism and the EIS.

When the Diamond economy's interest rate is low $\left(f^{\prime}\left(k^{D}\right)<n\right)$, there exists a steady state with bubble under one of two scenarios: (1) the EIS $\varepsilon \leq 1$ and the ascendant altruism $y$ is low enough (in this case, the value of bubble is decreasing in $y)$, and (2) the EIS $\varepsilon>1$ and the ascendant altruism $y$ is either low enough or high enough. In the first scenario, when the EIS $\varepsilon \leq 1$, the total savings $n k+b$ is decreasing and the modified interest rate $\mathcal{R}(y)$ is increasing in the ascendant altruism degree $y$. Since bubbles are financed through savings, the altruism degree should not be too high in order to ensure that the value of bubble is positive. In this case, we have $\mathcal{R}(y)<n$.

We now look at the case where $f^{\prime}\left(k^{D}\right)<n$, the EIS $\varepsilon>1$ and $y$ is high enough. When the EIS $\varepsilon>1$, the modified interest rate $\mathcal{R}(y)$ may not be monotonic in $y$ (because ascendant altruism generates two effects: the income when young is decreasing in $y$ but the income when old is increasing in $y$ ). However, it is decreasing in $y$ when $y$ is high enough (in the sense that $y>\bar{y}$ ). In this case, both the total savings and the value of bubble are increasing in the ascendant altruism degree $y$. So, $y$ must be higher some threshold in order to ensure that people spend a strictly positive amount in the bubble asset. It should be noticed that, in some cases, the modified interest rate $\mathcal{R}(y)$ may be lower than $n$ for any $y>0$, which means that there is a bubble for any $y>0$.

Interestingly, point 2.b of Proposition 1 indicates that we may have a steady state with bubble even the interest rate of the Diamond economy $f^{\prime}\left(k^{D}\right)$ is higher than $n$ (i.e., $k^{D}<k^{n}$ under-accumulation condition). This is an important difference between our model and that in (Tirole, 1985). The basic reason is from the fact that the value of bubble in our model is determined by the modified Euler equation (10d), according to which the total savings may not be monotonic in $y$. When the EIS $>1, f^{\prime}\left(k^{D}\right)>n$, and $y$ is high enough, the modified interest rate $\mathcal{R}(y)$ is lower than $n$, which ensures the existence of bubble.

\subsection{Transitional dynamics in a tractable case}

We now assume that $u(c)=\ln (c)$ and $f\left(k_{t}\right)=A k_{t}^{\alpha}$. Under this specification, we will show that the equilibrium sequences of capital and bubble will be explicitly computed. Indeed, conditions (10b), (10d) become

$$
\begin{aligned}
\frac{w\left(k_{t+1}\right)-\left(b_{t+1}+n k_{t+2}\right)}{\beta w\left(k_{t}\right)-(1+\beta)\left(b_{t}+n k_{t+1}\right)} & =\frac{1+y}{y} \frac{f^{\prime}\left(k_{t+1}\right)}{n} \\
\frac{f^{\prime}\left(k_{t+1}\right)}{n} & =\frac{b_{t+1}}{b_{t}} .
\end{aligned}
$$

To present our result, let us introduce some notations:

$$
\lambda_{ \pm} \equiv \frac{\mathfrak{b} \pm \sqrt{\mathfrak{b}^{2}-4 \mathfrak{a}}}{2}, \quad z \equiv \frac{\alpha(1+\beta+\beta y)}{\beta-\alpha-2 \alpha \beta-y(1-\beta-\alpha+2 \alpha \beta)},
$$


where $\mathfrak{a} \equiv \frac{1-\alpha}{\alpha} \frac{1+y}{y} \beta, \mathfrak{b} \equiv \frac{1-\alpha}{\alpha}+\frac{1+y}{y}(1+\beta)$.

Under above specifications, we can fully compute and characterize the set of equilibria.

Proposition 2. Assume that $u(c)=\ln (c)$ and $f(k)=A k^{\alpha}$.

1. There exists an equilibrium converging to a positive bubble if and only if

$$
\begin{aligned}
& \left.\frac{\alpha}{1-\alpha}<\frac{\beta}{1+\beta} \text { (or, equivalently, } f^{\prime}\left(k^{D}\right)<n\right) \\
& \text { and } y<y^{*} \equiv \frac{\beta-\alpha(1+2 \beta)}{1-\beta-\alpha(1-2 \beta)},
\end{aligned}
$$

where $k^{D}$ is the capital stock at the steady state of the Diamond economy (i.e., the economy without neither asset nor altruism). ${ }^{11}$ In this case, such an equilibrium is unique and balanced:

$$
\begin{aligned}
\left(k_{t+1}, b_{t}\right)_{t=0}^{\infty} & =\left(k_{t+1}, \frac{1}{z} n k_{t+1}\right)_{t=0}^{\infty} \\
k_{t} & =\rho_{1}^{\frac{1-\alpha}{1-\alpha}} k_{0}^{\alpha^{t}}
\end{aligned}
$$

where $\rho_{1} \equiv \alpha A / n$.

2. A bubbly equilibrium converges to a bubbleless steady state $(b=0)$ if and only if $z_{1}-z>\lambda_{-}\left(z_{0}-z\right)$ where $z_{t} \equiv n k_{t+1} / b_{t}$ is the portfolio sharing. In this case, since $z_{0}$ and $z_{1}$ are independently non-predetermined variables, the equilibrium path is indeterminate as well.

Proof. See Appendix.

This result is in line with point 1 of Proposition 1. Indeed, the existence of bubble requires two conditions: a low interest rate (capital overaccumulation) and a moderate ascendant altruism. The gain from explicit fundamentals (logarithmic utility and Cobb-Douglas production function) is the closed forms for equilibrium sequences enabling us to compute the impact of preference and technology parameters on capital intensity and bubble at any date. Even though the limit of capital intensity (20b) is independent of the altruism degree $y, y$ affects the asset value $b_{t}$. We observe that indeterminacy of portfolio sharing entails the equilibrium indeterminacy. Equilibrium indeterminacy depends on the true nature of asset. It is not an incidental effect but a robust equilibrium property.

Our result is related to Proposition 4 in Bosi et al. (2018b). The difference is that the equilibrium system in the model with descendant altruism of Bosi et al. (2018b) may be reduced to a one-dimensional system while it is two-dimensional in our model with ascendant altruism

Considering (20a) and (20b), and deriving with respect to $(\beta, y)$, we have the following result.

\footnotetext{
${ }^{11}$ In the Diamond economy $\left(a_{t}=h_{t}=0\right)$, the dynamics of capital becomes $k_{t+1}=\frac{\beta(1-\alpha)}{n(\beta+1)} A k_{t}^{\alpha}$. So, $k^{D}$ is determined by $1=\frac{\beta(1-\alpha)}{\alpha(\beta+1)} \frac{f^{\prime}\left(k^{D}\right)}{n}$. Therefore, condition $\frac{\alpha}{1-\alpha}<\frac{\beta}{1+\beta}$ is equivalent to $f^{\prime}\left(k^{D}\right)<n$.
} 
Corollary 1 (role of ascendant altruism). Along the equilibrium in point 1 of Proposition 2 , we find that

$$
\frac{\partial k_{t+1}}{\partial \beta}=\frac{\partial k_{t+1}}{\partial y}=0 \text { and } \frac{\partial b_{t}}{\partial \beta}>0, \frac{\partial b_{t}}{\partial y}<0, \forall t \geq 0 .
$$

As seen above, in the case of a larger ascendant altruism, individuals give $h_{t}$ to parents to finance their consumption. Their disposable income reduces to $w_{t}-h_{t}$ and savings lower as well through the bubble $b_{t}$

The size of bubble increases in the degree of patience $\beta$ at any date $t$ : indeed, if consumers care more about future, they save more and buy more bubble.

In the case of logarithmic utility function, the following result shows that we can explicitly compute the value of bubble.

Corollary 2 (bubbly steady state). Assume that $u(c)=\ln (c), f\left(k_{t}\right)=A k_{t}^{\alpha}$, and $\frac{\alpha}{1-\alpha}<\frac{\beta}{1+\beta}$. The existence of a bubbly steady state requires $f^{\prime}(k)=n$ and the size of bubble is given by

$$
b=n k\left[\frac{1-\alpha}{\alpha} \frac{\beta(1+y)-y}{(1+\beta)(1+y)-y}-1\right] .
$$

In addition, we have that $\frac{\partial b}{\partial \beta}>0$ and $\frac{\partial b}{\partial y}<0$.

Proof. See Appendix.

\section{Ascendant altruism, endogenous growth and bub- ble}

One of the simplest endogenous growth model is Romer (1986) where growth is driven by positive productive externalities. Assume that technology is represented by an augmented Cobb-Douglas production function

$$
F\left(K_{t}, L_{t}\right) \equiv A_{t} K_{t}^{\alpha} L_{t}^{1-\alpha}
$$

where the Total Factor Productivity $A_{t} \equiv A \bar{k}_{t}^{1-\alpha}$ changes over time and depends on productive externalities. These externalities mainly captures knowledge spillovers. Knowledge is proxied by the average capital intensity $\bar{k}_{t} . K_{t}$ and $L_{t}$ are the aggregate capital and the labor forces. Profit maximization implies that

$$
R_{t}=\alpha A \bar{k}_{t}^{1-\alpha} k_{t}^{\alpha-1}=\alpha A \equiv R
$$

and $w_{t}=(1-\alpha) A \bar{k}_{t}^{1-\alpha} k_{t}^{\alpha}=(1-\alpha) A k_{t}$.

In this case, the key variable becomes the growth factor of capital intensity: $g_{t} \equiv$ $k_{t+1} / k_{t}$, now endogenous. We notice that $g_{t}$ inherits from $k_{t+1}$ the quality of nonpredetermined variable at time $t$.

Proposition 3 (equilibrium uniqueness). Consider an endogenous growth model as above. Assume that $\alpha<1 / 2$. Let the elasticity of intertemporal substitution $\varepsilon$ be 
constant. Under ascendant altruism, the BGP is the unique equilibrium, with constant ratios:

$$
\begin{aligned}
\frac{b_{t}}{k_{t}} & =\frac{(1-2 \alpha) \gamma-\alpha A[\alpha+(1-\alpha) y]}{\alpha+\gamma / A} \\
\frac{b_{t}}{c_{t}} & =\frac{(1-2 \alpha) \gamma-\alpha A[\alpha+(1-\alpha) y]}{\alpha(1-\alpha) A}
\end{aligned}
$$

where $\gamma$ is defined by

$$
\gamma \equiv[A \alpha \beta(1+y)]^{\varepsilon}
$$

The capital intensity growth factor remains constant over time: $g_{t}=R / n=\alpha A / n$.

Proof. See Appendix.

Corollary 3. The existence of bubble $\left(b_{t}>0\right)$ requires that

$$
(1-2 \alpha)(A \alpha)^{\varepsilon-1} \beta^{\varepsilon}(1+y)^{\varepsilon}>\alpha+(1-\alpha) y .
$$

Proposition 3 and Corollary 3 lead to several implications. First, the existence of bubble requires a condition on fundamental, including the ascendant altruism degree. The intuition is in line with the case of exogenous growth that we have shown in Proposition 2. Let us focus here on two particualar cases.

- When the utility function is logarithmic (i.e., $\varepsilon=1$ ), this condition becomes

$$
y<y^{*} \equiv \frac{\beta-\alpha(1+2 \beta)}{1-\beta-\alpha(1-2 \beta)} .
$$

i.e., the degree of ascendant altruism must be moderate.

- However, when $\varepsilon>1$, condition (27) is satisfied if $y$ is high enough and the productivity $A$ is high. It means that when the technology is good enough, there may exist bubble even the ascendant altruism is strong. The intuition is similar to those in Proposition 1.

Second, our setup of endogenous growth allows us to examine the effects of altruism on the BGP. Indeed, according to (24) and (25), we can easily compute that

$$
\frac{\partial}{\partial y}\left(\frac{b_{t}}{k_{t}}\right)=\alpha(1-\alpha) \frac{(\varepsilon-1) \gamma-\alpha A}{(\alpha+\gamma / A)^{2}} \text { and } \frac{\partial}{\partial y}\left(\frac{b_{t}}{c_{t}}\right)=\frac{\varepsilon}{\alpha A} \frac{1-2 \alpha}{1-\alpha} \frac{\gamma}{1+y}-1
$$

and therefore obtain the following result.

Corollary 4 (Role of ascendant altruism). Let assumptions in Proposition 3 be satisfied. We have that

$$
\begin{aligned}
\frac{\partial}{\partial y}\left(\frac{b_{t}}{k_{t}}\right) & <0 \Leftrightarrow(\varepsilon-1)[A \alpha \beta(1+y)]^{\varepsilon}<\alpha A \\
\frac{\partial}{\partial y}\left(\frac{b_{t}}{c_{t}}\right) & <0 \Leftrightarrow \frac{\varepsilon}{\alpha A} \frac{1-2 \alpha}{1-\alpha}(A \alpha \beta)^{\varepsilon}(1+y)^{\varepsilon-1}<1 .
\end{aligned}
$$


Let us focus on the first point of Corollary 4. It shows how the relative size of bubble $\left(b_{t} / k_{t}\right)$ depends on fundamentals. In particular, the relative size of bubble is decreasing in the ascendant altruism degree (i.e., $\frac{\partial}{\partial y}\left(\frac{b_{t}}{k_{t}}\right)<0$ ) in the case of logarithmic preferences $(\varepsilon=1)$ or dominant income effects $(\varepsilon<1)$. In these cases, ascendant altruism reduces not only the savings (because gifts to parents are proportional to consumption of young), but also the bubble share in total savings (relative size of the bubble). We recover, even in the case of a pure bubble, the result with exogenous growth of Corollary 1.

However, the relative size of bubble may increase in $y$. Indeed, this happen if and only if $(\varepsilon-1) \beta^{\varepsilon}(1+y)^{\varepsilon}(\alpha A)^{\varepsilon-1}>1$ (this requires that $\varepsilon>1$, the ascendant altruism degree and the productivity $A$ are high enough).

\section{Conclusion}

We have investigate the interplay between ascendant altruism, pure bubble asset and economic dynamics in OLG models with both exogenous and endogenous growth.

In our exogenous growth framework, along the steady state equilibrium with bubble, the modified interest rate must be lower than the population growth rate $n$ but the benchmark interest rate of the Diamond economy $f^{\prime}\left(k^{D}\right)$ may be lower or higher than $n$. When the elasticity of intertemporal substitution (EIS) is lower than 1 , the existence of bubble requires that $f^{\prime}\left(k^{D}\right)<n$ and the ascendant altruism is moderate (in this case, the value of bubble is decreasing in the ascendant altruism degree. However, when the elasticity of intertemporal substitution (EIS) is higher than 1, the existence of bubble does not necessarily require that $f^{\prime}\left(k^{D}\right)<n$. Moreover, the value of bubble may be increasing (resp., decreasing) in the ascendant altruism if the latter is high enough (resp., low enough). Moreover, with logarithmic utility function and CobbDouglas production function, we have computed the explicit trajectories for capital and bubble.

In an endogenous growth model with Cobb-Douglas production function and constant EIS, we have explicitly computed the balanced growth path. We have also found that the existence and relative size of bubble depend strongly on the EIS and the ascendant altruism, which is consistent with the insights in the exogenous growth framework.

\section{Appendix: Formal Proofs}

Proof of Lemma 1. There exists a steady state with bubble if and only if the equation (12) has a solution in the interval $\left(0, w^{n}-n k^{n}\right)$. 
Necessary condition. First, $f\left(k^{n}\right)-2 n k^{n}>0$ is equivalent to $w\left(k^{n}\right)-n k^{n}>0$. Second, we observe that

$$
\begin{aligned}
u^{\prime}\left(\frac{w^{n}-n k^{n}}{1+y}\right) & <u^{\prime}\left(\frac{w^{n}-n k^{n}-b}{1+y}\right), \\
u^{\prime}\left(f^{\prime}\left(k^{n}\right)\left(n k^{n}+b\right)+n y \frac{w^{n}-n k^{n}-b}{1+y}\right) & =u^{\prime}\left(n f^{\prime}\left(k^{n}\right) k^{n}+n y \frac{w^{n}-n k^{n}}{1+y}+\frac{n b}{1+y}\right) \\
& \leq u^{\prime}\left(n f^{\prime}\left(k^{n}\right) k^{n}+n y \frac{w^{n}-n k^{n}}{1+y}\right) .
\end{aligned}
$$

By combining these inequalities with (12), we get (13b).

Sufficient condition. Suppose that (13a) and (13b) hold. Equation (12) is equivalent to

$$
u^{\prime}\left(\frac{w^{n}-n k^{n}-b}{1+y}\right)=(1+y) \beta n u^{\prime}\left(n^{2} k^{n}+n y \frac{w^{n}-n k^{n}}{1+y}+\frac{n b}{1+y}\right) .
$$

The left hand side is strictly increasing while the right hand side is strictly decreasing in $b$. So, condition (13b) ensures that equation (12) has a solution $b$ in the interval $\left(0, w^{n}-n k^{n}\right)$.

Proof of Proposition 1. To simplify notations, denote $w^{n} \equiv w\left(k^{n}\right)$.

Since $u^{\prime}(c)=c^{-\frac{1}{\varepsilon}}, \forall c$, the equation (12) becomes

$$
\begin{gathered}
\left(\frac{w^{n}-n k^{n}-b}{1+y}\right)^{-\frac{1}{\varepsilon}}=(1+y) \beta n\left(n\left(n k^{n}+b\right)+n y \frac{w^{n}-n k^{n}-b}{1+y}\right)^{-\frac{1}{\varepsilon}} \\
\Leftrightarrow\left(\frac{\left(n k^{n}+b\right)+y \frac{w^{n}-n k^{n}-b}{1+y}}{w^{n}-n k^{n}-b}\right)^{\frac{1}{\varepsilon}}=\beta n^{1-\frac{1}{\varepsilon}}(1+y)^{1-\frac{1}{\varepsilon}} \\
\Leftrightarrow \frac{w^{n}}{w^{n}-n k^{n}-b}-\frac{1}{1+y}=\beta^{\varepsilon} n^{\varepsilon-1}(1+y)^{\varepsilon-1} .
\end{gathered}
$$

So, the value of bubble is determined by the equation

$$
\frac{w^{n}}{w^{n}-n k^{n}-b}=\frac{1}{1+y}+\beta^{\varepsilon} n^{\varepsilon-1}(1+y)^{\varepsilon-1}
$$

Let $h(y)=\frac{1}{1+y}+\beta^{\varepsilon} n^{\varepsilon-1}(1+y)^{\varepsilon-1}$. We observe that : $b>0$ (a bubble exists) if and only if $h(y)>\frac{w^{n}}{w^{n}-n k^{n}}$.

We will prove the following claim: $h(0)>\frac{w^{n}}{w^{n}-n k^{n}}$ is equivalent to $k^{n}<k^{D}$ (or, equivalently, $\left.f^{\prime}\left(k^{D}\right)<n\right)$. Indeed, $h(0)>\frac{w^{n}}{w^{n}-n k^{n}}$ means that

$$
\frac{w^{n}}{w^{n}-n k^{n}}<1+\beta^{\varepsilon} n^{\varepsilon-1} \text {. }
$$

This is equivalent to

$$
\frac{\left(w\left(k^{n}\right)-n k^{n}\right)^{-\frac{1}{\varepsilon}}}{\beta f^{\prime}\left(k^{n}\right)\left(n f^{\prime}\left(k^{n}\right) k^{n}\right)^{-\frac{1}{\varepsilon}}}<1=\frac{\left(w\left(k^{D}\right)-n k^{D}\right)^{-\frac{1}{\varepsilon}}}{\beta f^{\prime}\left(k^{D}\right)\left(n f^{\prime}\left(k^{D}\right) k^{D}\right)^{-\frac{1}{\varepsilon}}} .
$$


Since the function $\frac{f(k)}{k f^{\prime}(k)}$ is decreasing in $k$, the function $\frac{(w(k)-n k)^{-\frac{1}{\varepsilon}}}{\beta f^{\prime}(k)\left(f^{\prime}(k) k\right)^{-\frac{1}{\varepsilon}}}$ is strictly increasing in $k$. So, condition (A.5) means that $k^{n}<k^{D}$, or, equivalently, $f^{\prime}\left(k^{D}\right)<n$.

We now consider two cases: $\varepsilon \leq 1$ and $\varepsilon>1$.

1. Consider the case $\varepsilon \leq 1$. It is easy to see that the function $h$ is decreasing in $y$. Hence, if $h(0) \leq \frac{w^{n}}{w^{n}-n k^{n}}$, equation (A.3) does not have positive solution $b$. To ensure that the value of bubble is positive, we must have $h(0)>\frac{w^{n}}{w^{n}-n k^{n}}$, which is $f^{\prime}\left(k^{D}\right)<n$. Under this condition, there is a unique solution, denoted by $\hat{y}$, to $h(y)=\frac{w^{n}}{w^{n}-n k^{n}}$. The existence of bubbly steady state is equivalent to $y<\hat{y}$.

2. Now, consider the case $\varepsilon>1$. Calculus gives

$$
h^{\prime}(y)=\frac{1}{(1+y)^{2}} \times\left(-1+(\varepsilon-1) \beta^{\varepsilon} n^{\varepsilon-1}(1+y)^{\varepsilon}\right) .
$$

Denote by $\tilde{y}$ the unique solution to $h^{\prime}(y)=0$. Precisely, $\tilde{y}=\frac{\left(\frac{1}{\varepsilon-1}\right)^{\frac{1}{\varepsilon}}}{\beta n^{1-\frac{1}{\varepsilon}}}-1$.

First, we consider the case $\tilde{y}>0$. The function $h$ is strictly decreasing on $(0, \tilde{y})$ and strictly increasing on $(\tilde{y}, \infty)$. The value $h(\tilde{y})$ is the minimum value of the function $h$.

(a) Consider the case $\frac{w^{n}}{w^{n}-n k^{n}}<h(0)$ (or, equivalently, $f^{\prime}\left(k^{D}\right)<n$ ).

i. If $h(\tilde{y})>\frac{w^{n}}{w^{n}-n k^{n}}$, then $h(y)>\frac{w^{n}}{w^{n}-n k^{n}}, \forall y>0$. In this case, take $\underline{y}=\bar{y}=0$.

ii. If $h(\tilde{y})=\frac{w^{n}}{w^{n}-n k^{n}}$, then $h(y)>\frac{w^{n}}{w^{n}-n k^{n}}, \forall y \neq \tilde{y}$. So, take $\underline{y}=\bar{y}=\tilde{y}$.

iii. If $h(\tilde{y})<\frac{w^{n}}{w^{n}-n k^{n}}$. Let $\underline{y}<\bar{y}$ be the two solutions to equation $h(y)=$ $\frac{w^{n}}{w^{n}-n k^{n}}$. Obviously, $\underline{y}<\tilde{y}<\bar{y}$. For $0<y<\underline{y}$ and for $y>\bar{y}$, we have $h(y)>\frac{w^{n}}{w^{n}-n k^{n}}$.

The bubble is positive if $y<y$ or $y>\bar{y}$. The function $h$ is decreasing on $(0, y)$ and increasing on $(\bar{y}, \infty)$. By equation (A.3), the value of bubble is decreasing on $(0, \underline{y})$ and increasing on $(\bar{y}, \infty)$.

(b) Consider the case $\frac{w^{n}}{w^{n}-n k^{n}}>h(0)$ (or, equivalently, $f^{\prime}\left(k^{D}\right)>n$ ). This implies $h(y)<\frac{w^{n}}{w^{n}-n k^{n}}$ for every $0 \leq y<\tilde{y}$. Let $\bar{y}$ be the unique solution to $h(y)=\frac{w^{n}}{w^{n}-n k^{n}}$. We have that $h(y)>\frac{w^{n}}{w^{n}-n k^{n}}$ (i.e., the bubbly existence condition) is equivalent to $y>\bar{y}$. Obviously, $\bar{y}>\tilde{y}$.

Since on $(\bar{y}, \infty)$, the function $h$ is increasing in respect to $y$, the value of bubble, determined by equation (A.3), is also increasing in $y$.

Second, we consider the case $\tilde{y} \leq 0$, then the function $h$ is strictly increasing in $(0, \infty)$ and takes any value in the interval $(h(0), \infty)$. So, claims 2.a and 2.b in Proposition 1 hold. Indeed, consider two sub-cases. (a) If $h(0)>\frac{w^{n}}{w^{n}-n k^{n}}$, then there is a steady state with bubble $(b>0)$ for any $y>0$. We can choose $\underline{y}=\bar{y}=0$ and obtain claim 2.a. (b) If $h(0)<\frac{w^{n}}{w^{n}-n k^{n}}$. Let $\bar{y}$ be the unique solution to $h(y)=\frac{w^{n}}{w^{n}-n k^{n}}$ and we obtain claim 2.b. 
Proof of Proposition 2. Since $\frac{b_{t+1}}{b_{t}}=\frac{f^{\prime}\left(k_{t+1}\right)}{n}=\frac{\alpha A k_{t+1}^{\alpha-1}}{n}$, we find that

$$
\frac{w\left(k_{t+1}\right)}{b_{t+1}}=\frac{(1-\alpha) A k_{t+1}^{\alpha}}{b_{t+1}}=\frac{1-\alpha}{\alpha} \frac{n k_{t+1}}{b_{t}} .
$$

Denote $z_{t} \equiv s_{t} / b_{t}=n k_{t+1} / b_{t}$ the portfolio sharing. The system (17a)-(17b) simplifies

$$
z_{t+2}=\left[\frac{1-\alpha}{\alpha}+\frac{1+y}{y}(1+\beta)\right] z_{t+1}-\frac{1-\alpha}{\alpha} \frac{1+y}{y} \beta z_{t}+\frac{1+y}{y}(1+\beta)-1 .
$$

The system (A.7) writes equivalently,

$$
\left[\begin{array}{c}
\zeta_{t+1}-z \\
z_{t+1}-z
\end{array}\right]=\left[\begin{array}{cc}
\mathfrak{b} & -\mathfrak{a} \\
1 & 0
\end{array}\right]\left[\begin{array}{c}
\zeta_{t}-z \\
z_{t}-z
\end{array}\right]
$$

where $\zeta_{t} \equiv z_{t+1}$ and $z$ is given by (18a).

Suppose that there exists an equilibrium converging to a steady state equilibrium with bubble. First, since $f(k)=A k^{\alpha}, \forall k>0$, and $f^{\prime}\left(k^{n}\right)=n$, condition $w\left(k^{n}\right)-$ $n k^{n}>0$ becomes $\alpha<0.5$. We observe that $z$ and bubble are positive if and only if $\beta-\alpha-2 \alpha \beta>y(1-\beta-\alpha+2 \alpha \beta)$. If $\beta-\alpha-2 \alpha \beta \leq 0$, we have $1-\beta-\alpha+2 \alpha \beta<0$. Thus, $0>\beta-\alpha-2 \alpha \beta+1-\beta-\alpha+2 \alpha \beta=1-2 \alpha$, a contradiction because $\alpha<0.5$. Therefore, we have $\beta-\alpha-2 \alpha \beta>0$. To sum up, we get (19a-19b). Notice that $\beta-\alpha-2 \alpha \beta>0$ implies $\alpha<0.5$.

Suppose now that (19a-19b) hold. Consider an equilibrium. We will prove that: if $z_{t} \equiv n k_{t+1} / b_{t}$ is bounded from above, then $z_{t}=z>0, \forall t \geq 0$.

According to (A.8), we obtain an explicit dynamics

$$
\left[\begin{array}{c}
\zeta_{t}-z \\
z_{t}-z
\end{array}\right]=\left[\begin{array}{cc}
\lambda_{-} & \lambda_{+} \\
1 & 1
\end{array}\right]\left[\begin{array}{cc}
\lambda_{-}^{t} & 0 \\
0 & \lambda_{+}^{t}
\end{array}\right]\left[\begin{array}{cc}
\lambda_{-} & \lambda_{+} \\
1 & 1
\end{array}\right]^{-1}\left[\begin{array}{c}
z_{1}-z \\
z_{0}-z
\end{array}\right]
$$

with eigenvalues given by (18a) or, more explicitly,

$$
z_{t}=z+\frac{\left(\lambda_{+}^{t}-\lambda_{-}^{t}\right)\left(z_{1}-z\right)+\left(\lambda_{+} \lambda_{-}^{t}-\lambda_{-} \lambda_{+}^{t}\right)\left(z_{0}-z\right)}{\lambda_{+}-\lambda_{-}} .
$$

We observe that $0<\mathfrak{b}^{2}-4 \mathfrak{a}<\mathfrak{b}^{2}$. Then, $0<\lambda_{-}<\mathfrak{b} / 2<\lambda_{+}<\mathfrak{b}$. Bubble positivity (that is $z>0$ ) requires

$$
\alpha<\frac{\beta}{1+2 \beta}<\frac{1}{3}
$$

which implies in turn that $\mathfrak{b}>2$. Hence, $\lambda_{+}>1$. In addition, $\lambda_{-}>1$ if and only if $1+\mathfrak{a}-\mathfrak{b}>0$, that is if and only if $z>0$.

Since $z_{t}$ and $\zeta_{t}$ are independently non-predetermined variable, the equilibrium with a persistent bubble exists (i.e., $z_{t}=z$, that is $s_{t}=n k_{t+1}=z b_{t}$ ), but it is unstable (if 
$z_{t} \neq z$ for some $\mathrm{t}$, the sequence $\left(z_{s}\right)_{s \geq t}$ diverges). Combining this and equation (17b), we have that

$$
\frac{f^{\prime}\left(k_{t+1}\right)}{n}=\frac{b_{t+1}}{b_{t}}=\frac{k_{t+2}}{k_{t+1}} .
$$

Thus, we find $k_{t+1}=\rho_{1} k_{t}^{\alpha}$ where $\rho_{1} \equiv \alpha A / n$ for any $t>0$.

Solving recursively, we get the solution (20a) with the explicit capital dynamics (20b).

However, $z_{t}=n k_{t+1} / b_{t}$ diverges to $+\infty$ if the bubble $b_{t}$ converges to 0 because the sequence $\left(k_{t}\right)$ is uniformly bounded from above. According to (A.9), this happens if and only if

$$
\begin{aligned}
+\infty & =\lim _{t \rightarrow \infty}\left[\left(\lambda_{+}^{t}-\lambda_{-}^{t}\right)\left(z_{1}-z\right)+\left(\lambda_{+} \lambda_{-}^{t}-\lambda_{-} \lambda_{+}^{t}\right)\left(z_{0}-z\right)\right] \\
& =\lim _{t \rightarrow \infty}\left[\lambda_{+}^{t}\left(z_{1}-z-\lambda_{-}\left(z_{0}-z\right)-\left[z_{1}-z-\lambda_{+}\left(z_{0}-z\right)\right]\left(\frac{\lambda_{-}}{\lambda_{+}}\right)^{t}\right)\right] \\
& =\left[z_{1}-z-\lambda_{-}\left(z_{0}-z\right)\right] \lim _{t \rightarrow \infty} \lambda_{+}^{t},
\end{aligned}
$$

that is if and only if $z_{1}-z>\lambda_{-}\left(z_{0}-z\right)$.

Proof of Corollary 2. According to (12), we have

$$
\frac{1+y}{w^{n}-n k^{n}-b}=\frac{(1+y) \beta n}{n\left(n k^{n}+b\right)+n y \frac{w^{n}-n k^{n}-b}{1+y}},
$$

or, equivalently, $\frac{w^{n}-\left(b+n k^{n}\right)}{\beta w^{n}-(1+\beta)\left(b+n k^{n}\right)}=\frac{1+y}{y}$. From this, we can easily compute (21).

We observe that $b>0$ if and only if $\beta-\alpha(1+2 \beta)-y(1-\alpha-\beta+2 \alpha \beta)>0$. Since we have $\beta-2 \alpha \beta>\alpha$ (because $\left.\frac{\alpha}{1-\alpha}<\frac{\beta}{1+\beta}\right)$ and $1-\alpha>(1-2 \alpha) \beta$, condition $b>0$ becomes $y<y^{*} \equiv \frac{\beta-\alpha(1+2 \beta)}{1-\beta-\alpha(1-2 \beta)}$.

Proof of Proposition 3. The productive side of the model does not change and profit maximization still yields $R_{t}=\alpha A \equiv R$ and $w_{t}=(1-\alpha) A k_{t}$.

Because of the no-arbitrage condition $\left(R_{t+1}=q_{t+1} / q_{t}\right)$, the household's program results in the following first-order conditions.

$$
\begin{aligned}
u^{\prime}\left(c_{t}\right) & =(1+y) \beta u^{\prime}\left(R n k_{t+1}+R b_{t}+n y c_{t+1}\right) R \\
(1-\alpha) A k_{t} & =(1+y) c_{t}+n k_{t+1}+b_{t} \\
b_{t+1} & =b_{t} \frac{R_{t+1}}{n}
\end{aligned}
$$

where $b_{t} \equiv q_{t} a_{t}$ with $a_{t}=a_{0} / n^{t}$.

Considering the isoelastic specification $u(x)=\frac{x^{1-1 / \varepsilon}}{1-1 / \varepsilon}$ and normalizing the system (A.11a)-(A.11c) by $b_{t}$, we get a two-dimensional dynamic system:

$$
\begin{aligned}
R u_{t+1}+y v_{t+1} & =\frac{\gamma}{R} v_{t}-1 \\
R u_{t+1} & =(1-\alpha) A u_{t}-(1+y) v_{t}-1
\end{aligned}
$$

where $u_{t} \equiv k_{t} / b_{t}$ and $v_{t} \equiv c_{t} / b_{t}$. 

obtain

The growth factor is given by $g_{t} \equiv \frac{k_{t+1}}{k_{t}}=\frac{R}{n} \frac{b_{t}}{k_{t}} \frac{k_{t+1}}{b_{t+1}}=\frac{R}{n} \frac{u_{t+1}}{u_{t}}$. In matrix terms, we

$$
\left[\begin{array}{l}
u_{t+1} \\
v_{t+1}
\end{array}\right]=\left[\begin{array}{cc}
\frac{1-\alpha}{\alpha} & -\frac{1+y}{A \alpha} \\
-\frac{A(1-\alpha)}{y} & \frac{1+y}{y}+\frac{\gamma}{\alpha A y}
\end{array}\right]\left[\begin{array}{l}
u_{t} \\
v_{t}
\end{array}\right]-\left[\begin{array}{c}
\frac{1}{\alpha A} \\
0
\end{array}\right] .
$$

The steady state is given by

$$
\left[\begin{array}{l}
u \\
v
\end{array}\right]=\frac{1}{(1-2 \alpha) \gamma-\alpha A[\alpha+(1-\alpha) y]}\left[\begin{array}{c}
\alpha+\frac{\gamma}{A} \\
\alpha(1-\alpha) A
\end{array}\right]
$$

provided that

$$
\gamma>\alpha A \frac{\alpha+(1-\alpha) y}{1-2 \alpha}
$$

We can compute the global dynamics:

$$
\left[\begin{array}{l}
u_{t} \\
v_{t}
\end{array}\right]=\left[\begin{array}{l}
u \\
v
\end{array}\right]+\left[\begin{array}{cc}
\frac{1-\alpha}{\alpha} & -\frac{1+y}{A \alpha} \\
-\frac{A(1-\alpha)}{y} & \frac{1+y}{y}+\frac{\gamma}{\alpha A y}
\end{array}\right]^{t}\left[\begin{array}{l}
u_{0}-u \\
v_{0}-v
\end{array}\right]
$$

with determinant and trace: $D=\frac{\gamma}{\alpha A y} \frac{1-\alpha}{\alpha}, T=\frac{1+y}{y}+\frac{\gamma}{\alpha A y}+\frac{1-\alpha}{\alpha}>0$.

The characteristic polynomial, evaluated in $-1,0$ and 1 , is given by

$$
\begin{aligned}
P(-1) & =1+T+D=\frac{1+y}{y}+\frac{1}{\alpha}\left(1+\frac{\gamma}{\alpha A y}\right)>0 \\
P(0) & =D=\frac{\gamma}{\alpha A y} \frac{1-\alpha}{\alpha}>0 \\
P(1) & =1-T+D=\frac{\gamma}{\alpha A y} \frac{1-2 \alpha}{\alpha}-\frac{1-2 \alpha}{\alpha}-\frac{1+y}{y} .
\end{aligned}
$$

Both the eigenvalues are real. Indeed,

$$
\begin{aligned}
T^{2}-4 D & =\left(\frac{1+y}{y}+\frac{\gamma}{\alpha A y}+\frac{1-\alpha}{\alpha}\right)^{2}-4 \frac{\gamma}{\alpha A y} \frac{1-\alpha}{\alpha} \\
& >\left(\frac{\gamma}{\alpha A y}+\frac{1-\alpha}{\alpha}\right)^{2}-4 \frac{\gamma}{\alpha A y} \frac{1-\alpha}{\alpha}=\left(\frac{\gamma}{\alpha A y}-\frac{1-\alpha}{\alpha}\right)^{2} \geq 0
\end{aligned}
$$

Since $\alpha<1 / 2$, we see that

$$
T=\frac{1-\alpha}{\alpha}+\frac{1+y}{y}+\frac{\gamma}{\alpha A y}>\frac{1-\alpha}{\alpha}+\frac{1+y}{y}>2
$$

and $\arg \min P(\lambda)=T / 2>1$. Then, the steady state is a saddle point if $P(1)<0$ and it is a source if $P(1)>0$.

It is easy to check that $P(1)>0$ iff $\gamma>\alpha A \frac{\alpha+(1-\alpha) y}{1-2 \alpha}$ which is exactly condition (A.12) for the positivity of the steady state. 


\section{References}

Axelrod, R., 1984. The Evolution of Cooperation. Basic Books, New York.

Barro, R.J., 1974. Are government bonds net wealth. Journal of Political Economy 82, 1095-1117.

Barro, R.J., Becker, G., 1989. Fertility choice in a model of economic growth. Econometrica 57, 481-501.

Becker, G.S. 1974. A theory of social interaction. Journal of Political Economy 82, 1063-1093.

Becker, G.S. 1976. Altruism, egoism, and genetic fitness: economics and sociobiology. Journal of Economic Literature 4, 817-826.

Becker, G.S., 1981. A Treatise on the Family. Harvard University Press, Cambridge.

Bewley, T., 1980. The optimal quantity of money. In: Kareken, J. H., Wallace, N. (Eds.) Models of Monetary Economics, Minneapolis: Federal Reserve Bank, 169-210.

Blanchard, O., and Fischer, S., 1989. Lectures on Macroeconomics. MIT Press, Cambridge.

Bosi, S., Le Van, C., Pham, N.-S., 2018a. Intertemporal equilibrium with heterogeneous agents, endogenous dividends and collateral constraints, Journal of Mathematical Economics 76, 1-20.

Bosi, S., Ha-Huy, T., Le Van, C., Pham, C.-T., and Pham, N.-S., 2018b. Financial bubbles and capital accumulation in altruistic economies. Journal of Mathematical Economics 75, 125-139.

Bosi, S., Ha-Huy, T., Pham, C.-T., Pham, N.-S., 2019. Rational bubbles in altruistic economies: when Tirole meets Ramsey. Economics Bulletin 39, 816-829.

Bosi, S., Le Van, C., Pham, N.-S., 2017. Asset bubbles and efficiency in a generalized two-sector model, Mathematical Social Sciences 88, 37-48

Bosi, S., Le Van, C., Pham, N.-S., 2021. Real indeterminacy and dynamics of asset price bubbles in general equilibrium. 〈halshs-02993656v2 . Link: https://halshs.archivesouvertes.fr/halshs-02993656v2

Bosi, S., Seegmuller, T., 2013. Rational bubbles and expectation-driven fluctuations. International Journal of Economic Theory 9, 69-83.

Brunnermeier, M.K., Oehmke, M., 2013. Bubbles, financial crises, and systemic risk. In: Constantinides, G. M., Harris, M., Stulz, R. M. (Eds.), Handbook of the Economics of Finance, vol. 2. Elsevier.

Cai, F., Giles, J., Meng, X., 2006. How well do children insure parents against low retirement income? An analysis using survey data from urban China. Journal of Public Economics, 90, 2229-2255. 
Clain-Chamosset-Yvrard, L, Seegmuller, T., 2019. Bubble on real estate: the role of altruism and fiscal policy. Studies in Nonlinear Dynamics \& Econometrics, Volume 23, Issue 4 .

Davies, J.B., 1981. Uncertain lifetime, consumption, and dissaving in retirement. Journal of Political Economy 89, 561-577.

Diamond, P., 1965. National debt in a neoclassical growth model. American Economic Review 55, 1126-1150.

Galperti, S., Strulovici, B., 2017. A theory of intergenerational altruism. Econometrica 85, 1175-1218.

Kazianga, H., 2006. Motives for household private transfers in Burkina Faso. Journal of Development Economics 79, 73-117.

Kocherlakota, N.R., 1992. Bubbles and constraints on debt accumulation. Journal of Economic Theory 57, 245-256.

Kotlikoff, L.J., Summers, L.H., 1981. The role of intergenerational transfers in aggregate capital accumulation. Journal of Political Economy 89, 706-732.

Laferrere, A., Wolff, F.-C., 2006. Microeconomic models of family transfers. In Kolm S.C., Ythier, J.M., (Eds.), Handbook of the Economics of Giving, Altruism and Reciprocity, Vol. 2. Elsevier.

Le Van, C., Pham, N.-S., 2016. Intertemporal equilibrium with financial asset and physical capital. Economic Theory 62, 155-199.

Martin, A., Ventura, J., 2018. The Macroeconomics of Rational Bubbles: A User's Guide. Annual Review of Economics, 10(1), 505-539. https://doi.org/10.1146/annurev-economics-080217-053534

Miao, J., 2014. Introduction to economic theory of bubbles. Journal of Mathematical Economics 53, p. 130-136.

Michel, F., Thibault, E., Vidal, J.-P., 2006. Intergenerational altruism and neoclassical growth models. In: Kolm, S.-C., Ythier, J. M., (Eds.), Handbook of the Economics of Giving, Altruism and Reciprocity, Vol. 2. Elsevier.

Montrucchio, L., 2004. Cass transversality condition and sequential asset bubbles. Economic Theory, 24, 645-663.

Nguyen, H.T., Liu, A.Y.C, Booth, A.L., 2012. Monetary Transfers from Children and the Labour Supply of Elderly Parents: Evidence from Vietnam. Journal of Development Studies 48, 1177-1191.

Park, C., 2014. Why do children transfer to their parents? Evidence from South Korea. Review of Economics of the Household 12, 461-485. 
Romer, P.M., 1986. Increasing returns and long-run growth. Journal of Political Economy $94,1002-1037$.

Santos, M. S., Woodford, M., 1997. Rational asset pricing bubbles. Econometrica, 65, $19-57$.

Smith, A., 1759. The Theory of Moral Sentiments. J. Bell and A. Kincaid, Edinburgh, and A. Millar, London.

Tirole, J., 1982. On the possibility of speculation under rational expectations. Econometrica 50, 1163-1181.

Tirole, J., 1985. Asset bubbles and overlapping generations. Econometrica 53, 14991528.

Weil, F., 1987. Confidence and the real value of money in an overlapping generations economy. Quarterly Journal of Economics, 102, 1-22.

Weil, F., 1990. On the possibility of price decreasing bubbles. Econometrica, 58, 14671474. 